\title{
Stabilization of Collective Motion in Three Dimensions: A Consensus Approach
}

\author{
Luca Scardovi, Naomi Ehrich Leonard, and Rodolphe Sepulchre
}

\begin{abstract}
This paper proposes a methodology to stabilize relative equilibria in a model of identical, steered particles moving in three-dimensional Euclidean space. Exploiting the Lie group structure of the resulting dynamical system, the stabilization problem is reduced to a consensus problem. We first derive the stabilizing control laws in the presence of allto-all communication. Providing each agent with a consensus estimator, we then extend the results to a general setting that allows for unidirectional and time-varying communication topologies.
\end{abstract}

\section{INTRODUCTION}

The problem of controlling the formation of a group of autonomous systems has received a lot of attention in recent years. This interest is principally due to the theoretical aspects that couple graph theoretic and dynamical systems concepts, and to the vast number of applications. Applications range from sensor networks, where a group of autonomous agents has to collect information about a process by choosing maximally informative samples [1], [2], to formation control of autonomous vehicles (e.g. unmanned aerial vehicles) [3], [4]. In these contexts it is relevant to consider the case where the ambient space is the threedimensional Euclidean space.

In the present paper we consider a model of identical particles moving at unit speed in three-dimensional Euclidean space and we address the problem of designing control laws to stabilize relative equilibria in the presence of limited communication among the agents. These equilibria are characterized by formations where the relative orientations and positions among the particles are constant [3]. They give rise to parallel, circular and helical formations. A motivating application for the present paper is the use of autonomous underwater vehicles to collect oceanographic measurements, extending the results in [1] to a three-dimensional setting. Furthermore the collective motions studied in the present paper have been empirically observed in swarming of animal groups [5].

The particles are subject to a gyroscopic feedback control which changes their orientation in space. The feedback is

This paper presents research results of the Belgian Network DYSCO (Dynamical Systems, Control, and Optimization), funded by the Interuniversity Attraction Poles Programme, initiated by the Belgian State, Science Policy Office. The scientific responsibility rests with its authors. The work is supported in part by ONR grants N00014-02-1-0826 and N00014-04$1-0534$.

L. Scardovi and R. Sepulchre are with the Department of Electrical Engineering and Computer Science, University of Liège, Belgium, 1.scardovi@ulg.ac.be, r.sepulchre@ulg.ac.be

N.E. Leonard is with the Department of Mechanical and Aerospace Engineering, Princeton University, naomi@ princeton.edu based only on the relative positions and the relative orientations of the particles and therefore preserves the $S E(3)$ symmetry of the formation. An important consequence is that no external reference is required. The desired control laws can be static or dynamic. A static control law requires to exchange only the relative positions and orientations among the particles at each time instant. Dynamic control laws additionally include a consensus variable that is shared with the communicating particles. The additional exchange of information is rewarded by an increased robustness with respect to communication failures (see [6] and [7] for details) and therefore is applicable to limited and time-varying communication scenarios.

The present paper generalizes, to three-dimensional space, earlier work in the plane [8], [9]. Preliminary results in $S E(3)$ have been presented in [10]. A similar approach applied to rigid body attitude synchronization has been presented in [11].

The rest of the paper is organized as follows. In Section II we define the kinematic model for a group of steered particles moving in three-dimensional Euclidean space with unitary velocity. In Section III we review some concepts from the theory of screws and we present a general methodology to stabilize relative equilibria on $S E(3)$. In Section IV we derive control laws that stabilize relative equilibria in the presence of all-to-all communication. Finally, in Section $\mathrm{V}$, we design dynamic control laws that stabilize relative equilibria in the presence of limited communication.

\section{A MOdel of STEERED PARTICLES IN $S E(3)$}

We consider a kinematic model of $N$ identical particles (with unitary mass) moving in three-dimensional Euclidean space at unit speed:

$$
\begin{aligned}
\dot{\boldsymbol{r}}_{k} & =\boldsymbol{x}_{k} \\
\dot{\boldsymbol{x}}_{k} & =\boldsymbol{u}_{k}^{a} \times \boldsymbol{x}_{k} \quad k=1,2, \ldots, N
\end{aligned}
$$

where $\boldsymbol{r}_{k} \in \mathbb{R}^{3}$ denotes the position of particle $k, \boldsymbol{x}_{k}$ is a unit-norm velocity vector and $\boldsymbol{u}_{k}^{a} \in \mathbb{R}^{3}$ is a control vector. Model (1) characterizes particles dynamics with forcing only in the directions normal to velocity, i.e. $\ddot{\boldsymbol{r}}_{k}=\boldsymbol{u}_{k}^{a} \times \dot{\boldsymbol{r}}_{k}$. An alternative to (1) is to provide each particle with an orthonormal frame and to write the system dynamics in a curve framing setting [3]:

$$
\begin{aligned}
& \dot{\boldsymbol{r}}_{k}=\boldsymbol{x}_{k} \\
& \dot{\boldsymbol{x}}_{k}=\boldsymbol{y}_{k} q_{k}+\boldsymbol{z}_{k} h_{k} \\
& \dot{\boldsymbol{y}}_{k}=-\boldsymbol{x}_{k} q_{k}+\boldsymbol{z}_{k} w_{k} \\
& \dot{\boldsymbol{z}}_{k}=-\boldsymbol{x}_{k} h_{k}-\boldsymbol{y}_{k} w_{k} \quad k=1, \ldots, N,
\end{aligned}
$$


where $\left(\boldsymbol{x}_{k}, \boldsymbol{y}_{k}, \boldsymbol{z}_{k}\right)$ is a right handed orthonormal frame associated to particle $k$ (in particular $\boldsymbol{x}_{k} \in S_{2}$ is the (unit) velocity vector). The scalars $q_{k}, h_{k}$ represent the curvature controls of the $k$ th particle. The scalar $w_{k}$ adds a further degree of freedom allowing rotations about the axis $\boldsymbol{x}_{k}$. In vector notation we define

$$
\boldsymbol{u}_{k}=\left[\begin{array}{c}
w_{k} \\
-h_{k} \\
q_{k}
\end{array}\right]
$$

The advantage of using model (2) instead of model (1) relies on its group structure. Model (2) indeed defines a control system on the Lie group $S E(3)$ and the dynamics (2) can be expressed in terms of the group variables $g_{k} \in S E(3)$ :

$$
\dot{g}_{k}=g_{k} \hat{\boldsymbol{\xi}}_{k}, \quad k=1, \ldots, N,
$$

where $\hat{\boldsymbol{\xi}}_{k} \in s e(3)$ is an element of the Lie algebra of $S E(3)$. From (2) we obtain

$$
g_{k}=\left[\begin{array}{cc}
R_{k} & \boldsymbol{r}_{k} \\
\mathbf{0} & 1
\end{array}\right], \quad R_{k}=\left[\boldsymbol{x}_{k}, \boldsymbol{y}_{k}, \boldsymbol{z}_{k}\right] \in S O(3),
$$

and

$$
\hat{\boldsymbol{\xi}}_{k}=\left[\begin{array}{cc}
\hat{\boldsymbol{u}}_{k} & \boldsymbol{e}_{1} \\
\mathbf{0} & 0
\end{array}\right]
$$

where

$$
\hat{\boldsymbol{u}}_{k}=\left[\begin{array}{ccc}
0 & -q_{k} & -h_{k} \\
q_{k} & 0 & -w_{k} \\
h_{k} & w_{k} & 0
\end{array}\right]
$$

is a skew-symmetric matrix that represents an element of the tangent space to $S O(3)$. We denote by $\left(\boldsymbol{e}_{1}, \boldsymbol{e}_{2}, \boldsymbol{e}_{3}\right)$ the standard orthonormal basis for $\mathbb{R}^{3}$.

When only the orientations of the particles are taken into account, the reduced dynamics of (4) are

$$
\dot{R}_{k}=R_{k} \hat{\boldsymbol{u}}_{k}, \quad k=1, \ldots, N
$$

and the system evolves on the Lie group $S O(3)$.

It is worth noting that the following relation exists between the control vector $\boldsymbol{u}_{k}^{a}$ in (1) and the vector $\boldsymbol{u}_{k}$ in (3)

$$
\boldsymbol{u}_{k}^{a}=R_{k} \boldsymbol{u}_{k}
$$

Therefore $\boldsymbol{u}_{k}^{a}$ can be interpreted as the control vector $\boldsymbol{u}_{k}$ expressed in the "absolute" reference frame.

If the curvature controls in model (2) are feedback functions of shape quantities (i.e. relative frame orientations and relative positions), the closed-loop vector field is invariant under an action of the symmetry group $S E(3)$. The resulting closed-loop dynamics evolve in a quotient manifold called shape space and the equilibria of the reduced dynamics are called relative equilibria. Relative equilibria of the model (2) have been characterized in [3]. The equilibria, depicted in Figure 1, are of three types:

i) Parallel motion: all particles move in the same direction with arbitrary relative positions;

ii) Circular motion: all particles draw circles with the same radius, in planes orthogonal to the same axis of rotation;

iii) Helical motion: all particles draw circular helices with the same radius, pitch, axis and axial direction of motion.

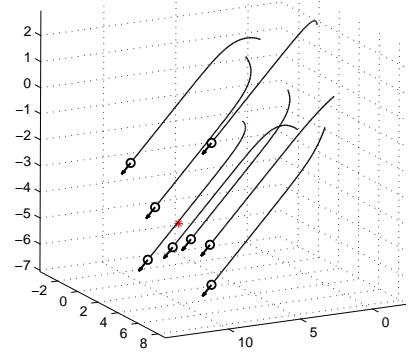

(a)

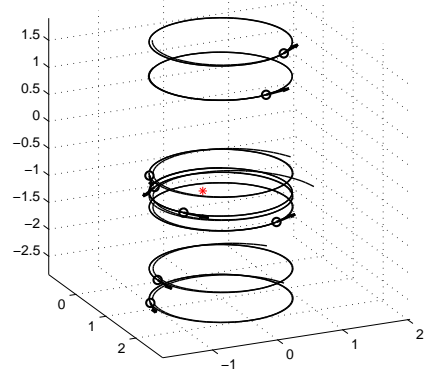

(b)

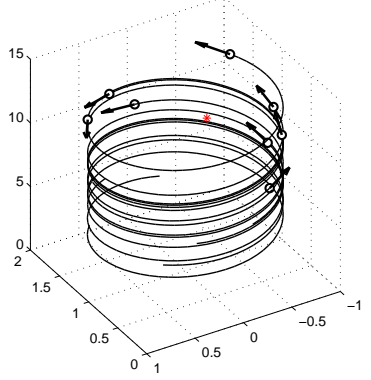

(c)

Fig. 1. The three types of relative equilibria: (a) parallel, (b) circular and (c) helical.

The present paper presents stabilizing feedback controls in shape space for the three types of relative equilibria.

\section{Stabilization OF RELATIVE EQUilibria AS A CONSENSUS PROBLEM}

In terms of screw theory [12], an element of $s e(3)$ is called a twist. The motion produced by a constant twist is called a screw motion. The operator denoted by $\vee$ extracts the 6 dimensional vector which parameterizes a twist: (5) yields

$$
\boldsymbol{\xi}_{k}=\left[\begin{array}{cc}
\hat{\boldsymbol{u}}_{k} & \boldsymbol{e}_{1} \\
\mathbf{0} & 0
\end{array}\right]^{\vee}=\left[\begin{array}{c}
\boldsymbol{e}_{1} \\
\boldsymbol{u}_{k}
\end{array}\right]
$$

The inverse operator, $\wedge$, expresses the twist in homogeneous coordinates starting from a vector form: (5) yields

$$
\hat{\boldsymbol{\xi}}_{k}=\left[\begin{array}{c}
\boldsymbol{e}_{1} \\
\boldsymbol{u}_{k}
\end{array}\right]^{\wedge}=\left[\begin{array}{cc}
\hat{\boldsymbol{u}}_{k} & \boldsymbol{e}_{1} \\
\mathbf{0} & 0
\end{array}\right]
$$


A twist $\boldsymbol{\xi}=\left[\boldsymbol{v}^{T}, \boldsymbol{\omega}^{T}\right]^{T} \in \mathbb{R}^{6}$ defines the screw motion $e^{\hat{\boldsymbol{\xi}}_{t}}$ on $S E(3)$ [12]. When $\boldsymbol{\omega} \neq 0$ this motion corresponds to a rotation by an amount $\theta=\|\boldsymbol{\omega}\|$ about the axis $\boldsymbol{l}$, followed by translation by an amount $p\|\boldsymbol{\omega}\|$ parallel to the axis $\boldsymbol{l}$. When $\boldsymbol{\omega}=0$ the corresponding screw motion consists of a pure translation along the axis of the screw by a distance $M=\|\boldsymbol{v}\|$. The relations among the screw $(\boldsymbol{l}, p, M)$ and twist are the following [12]:

$$
\begin{array}{r}
p= \begin{cases}\frac{\boldsymbol{\omega}^{T} \boldsymbol{v}}{\|\boldsymbol{\omega}\|^{2}}, & \text { if } \boldsymbol{\omega} \neq 0 \\
\boldsymbol{\omega}, & \text { if } \boldsymbol{\omega}=0\end{cases} \\
\boldsymbol{l}= \begin{cases}\frac{\boldsymbol{\omega} \times \boldsymbol{v}}{\|\boldsymbol{\omega}\|^{2}}+\lambda \boldsymbol{\omega}, & \text { if } \boldsymbol{\omega} \neq 0 \\
0+\lambda \boldsymbol{v}, & \text { if } \boldsymbol{\omega}=0\end{cases} \\
M= \begin{cases}\|\boldsymbol{\omega}\|, & \text { if } \boldsymbol{\omega} \neq 0 \\
\|\boldsymbol{v}\|, & \text { if } \boldsymbol{\omega}=0\end{cases}
\end{array}
$$

where $\lambda \in \mathbb{R}$. In the context of model (4), the twist (in body coordinates) is given by $\boldsymbol{\xi}_{k}=\left[\boldsymbol{e}_{1}^{T}, \boldsymbol{u}_{k}^{T}\right]^{T}$. To map $\boldsymbol{\xi}_{k}$ into a spatial reference frame, one uses the adjoint transformation associated with $g_{k}$

$$
\operatorname{Ad}_{g_{k}}=\left[\begin{array}{cc}
R_{k} & \hat{\boldsymbol{r}}_{k} R_{k} \\
0 & R_{k}
\end{array}\right]
$$

which yields

$$
\begin{aligned}
\boldsymbol{\xi}_{k}^{a} & =\operatorname{Ad}_{g_{k}} \boldsymbol{\xi}_{k}=\left[\begin{array}{c}
\boldsymbol{x}_{k}+\boldsymbol{r}_{k} \times R_{k} \boldsymbol{u}_{k} \\
R_{k} \boldsymbol{u}_{k}
\end{array}\right] \\
& =\left[\begin{array}{c}
\boldsymbol{x}_{k}+\boldsymbol{r}_{k} \times \boldsymbol{u}_{k}^{a} \\
\boldsymbol{u}_{k}^{a}
\end{array}\right] .
\end{aligned}
$$

To give a geometric interpretation to (8) we compute the relative screw coordinates (expressed in a global frame) and we obtain an (instantaneous) pitch

$$
p_{k}=\frac{\boldsymbol{e}_{1}^{T} \boldsymbol{u}_{k}}{\left\|\boldsymbol{u}_{k}\right\|^{2}}
$$

an (instantaneous) axis

$$
\boldsymbol{l}_{k}^{a}=\left\{\begin{array}{ll}
\boldsymbol{u}_{k}^{a} \times \frac{\boldsymbol{x}_{k}+\boldsymbol{r}_{k} \times \boldsymbol{u}_{k}^{a}}{\left\|\boldsymbol{u}_{k}\right\|^{2}}+\lambda \boldsymbol{u}_{k}^{a}, & \text { if } \boldsymbol{u}_{k} \neq 0 \\
0+\lambda \boldsymbol{x}_{k}, & \text { if } \boldsymbol{u}_{k}=0
\end{array},\right.
$$

and (instantaneous) magnitude

$$
M_{k}= \begin{cases}\left\|\boldsymbol{u}_{k}\right\|, & \text { if } \boldsymbol{u}_{k} \neq 0 \\ 1, & \text { if } \boldsymbol{u}_{k}=0 .\end{cases}
$$

Therefore, each constant control $\boldsymbol{u}_{k}$ defines a screw motion (corresponding to helical, circular or straight motion).

Consider two particles and their respective group variables $g_{k}$ and $g_{j}$. The dynamics for $g_{k j}=g_{k}^{-1} g_{j}$ (the shape variable) are given (see [3]) by

$$
\begin{aligned}
\dot{g}_{k j} & =-g_{k}^{-1} \dot{g}_{k} g_{k}^{-1} g_{j}+g_{k}^{-1} g_{j} \hat{\boldsymbol{\xi}}_{j} \\
& =-\hat{\boldsymbol{\xi}}_{k} g_{k j}+g_{k j} \hat{\boldsymbol{\xi}}_{j} \\
& =g_{k j}\left(\hat{\boldsymbol{\xi}}_{j}-\operatorname{Ad}_{g_{k j}^{-1}} \hat{\boldsymbol{\xi}}_{k}\right) .
\end{aligned}
$$

Equation (9) implies that a relative equilibrium of (4) is reached when the twists (expressed in the same reference frame) are equal for all the particles, i.e. $\boldsymbol{\xi}_{k}^{a}=\boldsymbol{\xi}_{0}^{a}$ for $k=1, \ldots, N, \boldsymbol{\xi}_{0}^{a}$ arbitrary. Moreover the screw coordinates of the common value $\boldsymbol{\xi}_{0}^{a}$ provide a geometrical description of the relative equilibria. Therefore the relative equilibria are characterized by a screw motion whose geometric parameters are a pitch, an axis and a magnitude uniquely determined by the consensus value $\boldsymbol{\xi}_{0}^{a}$. We summarize the above discussion as follows.

Proposition 1: The following statements are equivalent: i) System (2) is at a relative equilibrium.

ii) The twists $\boldsymbol{\xi}_{k}^{a}$ defined by (8) are equal for $k=1,2, \ldots, N$, i.e. the following algebraic condition is satisfied

$$
\tilde{\Pi} \xi^{a}=0
$$

where $\tilde{\Pi}=\left(I-\frac{1}{N} \mathbf{1 1}^{T}\right) \otimes I_{6}$ and $\boldsymbol{\xi}^{a}=\operatorname{col}\left(\boldsymbol{\xi}_{1}^{\mathrm{a}}, \boldsymbol{\xi}_{2}^{\mathrm{a}}, \ldots, \boldsymbol{\xi}_{\mathrm{N}}^{\mathrm{a}}\right)$.

Proposition 1 reduces the problem of stabilizing a relative equilibrium on $S E(3)$ to a consensus problem on twists.

Remark 1: The discussion above particularizes to $S E(2)$. The (planar) model

$$
\begin{aligned}
\dot{\boldsymbol{r}}_{k} & =\boldsymbol{x}_{k} \\
\dot{\boldsymbol{x}}_{k} & =u_{k} \boldsymbol{y}_{k} \\
\dot{\boldsymbol{y}}_{k} & =-u_{k} \boldsymbol{x}_{k}
\end{aligned}
$$

In the Lie group $S E(2)$, we obtain

$$
g_{k}=\left[\begin{array}{cc}
R_{k} & \boldsymbol{r}_{k} \\
0 & 1
\end{array}\right], \quad \hat{\boldsymbol{\xi}}_{k}=\left[\begin{array}{cc}
\hat{\boldsymbol{u}}_{k} & \boldsymbol{e}_{1} \\
0 & 0
\end{array}\right]
$$

where

$$
\begin{gathered}
R_{k}=\left[\boldsymbol{x}_{k}, \boldsymbol{y}_{k}\right] \in S O(2) \\
\hat{\boldsymbol{u}}_{k}=\left[\begin{array}{cc}
0 & -u_{k} \\
u_{k} & 0
\end{array}\right]=J u_{k}, \quad J=\left[\begin{array}{cc}
0 & -1 \\
1 & 0
\end{array}\right]
\end{gathered}
$$

and $\boldsymbol{e}_{1}=[1,0]^{T}$. In this case the twist is $\boldsymbol{\xi}_{k}=\left[\boldsymbol{e}_{1}^{T}, u_{k}\right]^{T} \in$ $\mathbb{R}^{3}$. By mapping the twist coordinates to a spatial frame we obtain

$$
\xi_{k}^{a}=\left[\begin{array}{c}
\boldsymbol{x}_{k}-u_{k} J \boldsymbol{r}_{k} \\
u_{k}
\end{array}\right]
$$

When $u_{k}$ is constant, only two types of motion are possible for (10), straight motion $\left(u_{k}=0\right)$ and circular motion $\left(u_{k}=\right.$ $\left.\omega_{0}\right)$. When (11) are equal and constant for all the particles the resulting motion is characterized by a parallel formation $\left(u_{k}=0\right)$ and a circular formation about the same point $\left(u_{k} \neq\right.$ 0 and constant). The control laws are derived in [8], [9].

\section{STABILIZATION OF RELATIVE EQUILIBRIA IN THE PRESENCE OF ALL-TO-ALL COMMUNICATION}

From (8), when a particle $k$ applies the constant control $\boldsymbol{u}_{k}=\boldsymbol{\omega}_{k}$, the (constant) twist expressed in the spatial reference frame is

$$
\boldsymbol{\xi}_{k}^{a}=\left[\begin{array}{c}
\boldsymbol{x}_{k}+\boldsymbol{r}_{k} \times R_{k} \boldsymbol{\omega}_{k} \\
R_{k} \boldsymbol{\omega}_{k}
\end{array}\right]=\left[\begin{array}{c}
\boldsymbol{s}_{k} \\
R_{k} \boldsymbol{\omega}_{k}
\end{array}\right] .
$$

Motivated by Prop. 1 a natural candidate Lyapunov function is

$$
V\left(\boldsymbol{\xi}^{a}\right)=\frac{1}{2}\left\|\tilde{\Pi} \boldsymbol{\xi}^{a}\right\|^{2}=\frac{1}{2} \sum_{k=1}^{N}\left\|\boldsymbol{\xi}_{k}^{a}-\boldsymbol{\xi}_{\mathrm{av}}^{a}\right\|^{2}
$$


where the subscript av is used to the note average quantities, i.e.

$$
\boldsymbol{\xi}_{\mathrm{av}}^{a}=\frac{1}{N} \sum_{k=1}^{N} \boldsymbol{\xi}_{k}^{a}
$$

This is the approach pursued in [8] for collective motion in $S E(2)$.

Unfortunately from (12) it is evident that the first component $s_{k}$ is not linear in the state variables. As a consequence $\boldsymbol{s}_{\mathrm{av}} \neq \boldsymbol{x}_{\mathrm{av}}+\boldsymbol{r}_{\mathrm{av}} \times \boldsymbol{\omega}_{\mathrm{av}}$ and the approach followed in [8] does not yield shape control laws. To overcome this obstacle we first stabilize the motion about an axis of rotation whose direction is fixed. In Section IV-B we relax the design by replacing, in the control laws, the fixed direction of the axis of rotation with (local) consensus variables, thereby obtaining stabilizing shape control laws.

\section{A. Stabilization about an axis of rotation with a fixed direc- tion}

Let $\boldsymbol{\omega} \in \mathbb{R}^{3}$ be a fixed constant vector. Observe that under the constant control law $\boldsymbol{\omega}_{k}=R_{k}^{T} \boldsymbol{\omega}$, a relative equilibrium is reached when the vectors $s_{k}$ in (12) are equal for all the particles.

Up to an additive constant the Lyapunov function (13) becomes

$$
S(\boldsymbol{s}, \boldsymbol{\omega})=\frac{1}{2} \sum_{k=1}^{N}\left\|\boldsymbol{s}_{k}-\boldsymbol{s}_{\mathrm{av}}\right\|^{2}
$$

where $\boldsymbol{s}_{k}=\boldsymbol{x}_{k}+\boldsymbol{r}_{k} \times \boldsymbol{\omega}$ and $\boldsymbol{s}=\left(\boldsymbol{s}_{1}^{T}, \ldots, \boldsymbol{s}_{N}^{T}\right)^{T}$. The time derivative is

$\dot{S}=\sum_{k=1}^{N}<\boldsymbol{s}_{k}-\boldsymbol{s}_{\mathrm{av}}, \dot{\boldsymbol{s}}_{k}>=\sum_{k=1}^{N}<\boldsymbol{s}_{k}-\boldsymbol{s}_{\mathrm{av}}, \dot{\boldsymbol{x}}_{k}+\boldsymbol{x}_{k} \times \boldsymbol{\omega}>$.

By making the last term explicit we obtain

$\dot{S}=\sum_{k=1}^{N}<\boldsymbol{s}_{k}-\boldsymbol{s}_{\mathrm{av}}, \boldsymbol{y}_{k}\left(q_{k}-<\boldsymbol{\omega}, \boldsymbol{z}_{k}>\right)+\boldsymbol{z}_{k}\left(h_{k}+<\boldsymbol{\omega}, \boldsymbol{y}_{k}>\right)>$.

The control law

$$
\begin{aligned}
& \boldsymbol{q}_{k}=<\boldsymbol{\omega}, \boldsymbol{z}_{k}>-<\boldsymbol{s}_{k}-\boldsymbol{s}_{\mathrm{av}}, \boldsymbol{y}_{k}> \\
& \boldsymbol{h}_{k}=-<\boldsymbol{\omega}, \boldsymbol{y}_{k}>-<\boldsymbol{s}_{k}-\boldsymbol{s}_{\mathrm{av}}, \boldsymbol{z}_{k}>
\end{aligned}
$$

results in a non-increasing $S$

$$
\dot{S}=-\sum_{k=1}^{N}\left(q_{k}-<\boldsymbol{\omega}, \boldsymbol{z}_{k}>\right)^{2}+\left(h_{k}+<\boldsymbol{\omega}, \boldsymbol{y}_{k}>\right)^{2} \leq 0 .
$$

By substituting the definition of $s_{k}$ in the control law (15) we obtain

$$
\begin{aligned}
& \boldsymbol{q}_{k}=\quad<\boldsymbol{\omega}, \boldsymbol{z}_{k}>-<\left(\boldsymbol{r}_{k}-\boldsymbol{r}_{\mathrm{av}}\right) \times \boldsymbol{\omega}-\boldsymbol{x}_{\mathrm{av}}, \boldsymbol{y}_{k}> \\
& \boldsymbol{h}_{k}=-<\boldsymbol{\omega}, \boldsymbol{y}_{k}>-<\left(\boldsymbol{r}_{k}-\boldsymbol{r}_{\mathrm{av}}\right) \times \boldsymbol{\omega}-\boldsymbol{x}_{\mathrm{av}}, \boldsymbol{z}_{k}>.
\end{aligned}
$$

The parameter $w_{k}$ in (2) is set to $\left\langle\boldsymbol{x}_{k}, \boldsymbol{\omega}\right\rangle$ to obtain a constant twist in steady state. By using the definition (3) the control law (17) can be written in the compact form

$$
\boldsymbol{u}_{k}=R_{k}^{T}\left(\boldsymbol{\omega}+\left[\left(\boldsymbol{r}_{k}-\boldsymbol{r}_{\mathrm{av}}\right) \times \boldsymbol{\omega}-\boldsymbol{x}_{\mathrm{av}}\right] \times \boldsymbol{x}_{k}\right)
$$

Note that the $s_{k}$ dynamics equipped with the control law (18) are

$$
\dot{\boldsymbol{s}}_{k}=-\Pi_{\boldsymbol{x}_{k}}\left(\boldsymbol{s}_{k}-\boldsymbol{s}_{\mathrm{av}}\right),
$$

where $\Pi_{\boldsymbol{x}_{k}}=I-\boldsymbol{x}_{k} \boldsymbol{x}_{k}^{T}$ is the projection matrix on the orthogonal complement of the subspace spanned by $\boldsymbol{x}_{k}$.

Theorem 1: Consider model (2) with the control law (18). All the solutions converge to an equilibrium of (19). The only asymptotically stable equilibria are relative equilibria of (2). Every other equilibrium of (19) is unstable.

Proof: From (16), $S$ is non-increasing along the solutions of (2) and, by the La Salle invariance principle all the solutions converge to the largest invariant set $\Gamma$ where

$$
\left(\boldsymbol{s}_{k}-\boldsymbol{s}_{\mathrm{av}}\right) \times \boldsymbol{x}_{k}=0,
$$

that characterizes the equilibria of (19). In this set $\dot{\boldsymbol{x}}_{k}=$ $\boldsymbol{\omega} \times \boldsymbol{x}_{k}$ and $\boldsymbol{s}_{k}$ is constant for $k=1, \ldots, N$. We divide the analysis in three parts to analyze $\Gamma$.

i) Suppose that in steady state $\boldsymbol{\omega} \times \boldsymbol{x}_{k} \neq 0$ for every $k$. Then (20) can hold only if $\boldsymbol{s}_{k}=\boldsymbol{s}_{0}$ for every $k$ and for some fixed $s_{0} \in \mathbb{R}^{3}$, this set defines a global minimum for $S$ and therefore is asymptotically stable. These equilibria correspond to circular or helical relative equilibria (with axis of rotation parallel to $\omega$ ).

ii) Suppose now that in steady state $\dot{\boldsymbol{x}}_{k}=\boldsymbol{\omega} \times \boldsymbol{x}_{k}=0$ for every $k$. From (20) we obtain

$$
\left(\boldsymbol{s}_{k}-\boldsymbol{s}_{\mathrm{av}}\right) \times \boldsymbol{\omega}=0
$$

for every $k$, which implies $\left(\boldsymbol{r}_{k}-\boldsymbol{r}_{\mathrm{av}}\right) \times \boldsymbol{\omega}=0$. Therefore in steady state the Lyapunov function (14) reduces to

$$
S=\frac{1}{2} \sum_{k=1}^{N}\left\|\boldsymbol{x}_{k}-\boldsymbol{x}_{\mathrm{av}}\right\|^{2}
$$

The equilibria are characterized by the vectors $\boldsymbol{x}_{k}, \quad k=$ $1, \ldots, N$, all parallel to the constant vector $\boldsymbol{\omega}$. Note that this configuration involves $N-K$ velocity vectors aligned to $\boldsymbol{\omega}$ and $K$ velocity vectors anti-aligned to $\boldsymbol{\omega}$ (or vice-versa), where $0 \leq K \leq \frac{N}{2}$. When $K=0$, potential (21) is zero (global minimum), and therefore the configuration defines asymptotically stable equilibria. These equilibria correspond to collinear formations (with the same direction of motion) parallel to $\boldsymbol{\omega}$.

When $K=\frac{N}{2}$, potential (21) attains a global maximum, and therefore the configuration defines unstable equilibria. Every other value of $K$ corresponds to a saddle point and is therefore unstable. To see this it is sufficient to express $\boldsymbol{x}_{k}$ and $\boldsymbol{\omega}$ in polar coordinates and to show that $S$ can decrease under an arbitrary small perturbation (see Theorem 1 in [10] for similar calculations).

iii) It remains to analyze the situation where $\boldsymbol{\omega} \times \boldsymbol{x}_{k} \neq 0$ for $k \in G_{1}$ and $\boldsymbol{\omega} \times \boldsymbol{x}_{j}=0$ for $j \in G_{2}$, where $G_{1}$ and $G_{2}$ denote two disjoint groups of particles such that $G_{1} \cup G_{2}=$ $\{1, \ldots, N\}$. In such a situation we obtain

$$
\begin{aligned}
& \boldsymbol{s}_{k}-\boldsymbol{s}_{\mathrm{av}}=0, \quad k \in G_{1} \\
& \left(\boldsymbol{s}_{j}-\boldsymbol{s}_{\mathrm{av}}\right) \times \boldsymbol{\omega}=0, \quad j \in G_{2} \text {, }
\end{aligned}
$$


where $s_{j} \neq s_{\mathrm{av}}, j \in G_{2}$. In this set the Lyapunov function (14) reduces to

$$
\begin{aligned}
S & =\frac{1}{2} \sum_{k \in G_{2}}\left\|\boldsymbol{s}_{k}-\boldsymbol{s}_{\mathrm{av}}\right\|^{2} \\
& =\frac{1}{2} \sum_{k \in G_{2}}\left\|<\boldsymbol{s}_{k}-\boldsymbol{s}_{\mathrm{av}}, \boldsymbol{x}_{k}>\boldsymbol{x}_{k}\right\|^{2} .
\end{aligned}
$$

Since $\boldsymbol{s}_{j} \neq \boldsymbol{s}_{\mathrm{av}}$, and $\boldsymbol{x}_{j}$ is parallel to $\boldsymbol{\omega}$ for every $j \in G_{2}$, we conclude from (23) that this set does not correspond to global minima of (14). It can be shown that under suitable perturbations of $\boldsymbol{x}_{k}, k \in G_{2}$ (such that $\boldsymbol{s}_{\mathrm{av}}$ is constant) (23) is decreasing and therefore conditions (22) define an unstable set (the details are omitted due to space constraints).

In steady state, the particles motion is characterized by a constant (consensus) twist $\boldsymbol{\xi}_{0}=\left[\boldsymbol{s}_{0}^{T}, \boldsymbol{\omega}^{T}\right]^{T}$. The corresponding screw parameters are a pitch $p_{0}=<\boldsymbol{s}_{0}, \boldsymbol{\omega}>/\|\boldsymbol{\omega}\|^{2}$, an axis $\boldsymbol{l}_{0}=\left\{\boldsymbol{s}_{0} \times \boldsymbol{\omega} /\|\boldsymbol{\omega}\|^{2}+\lambda \boldsymbol{\omega}, \lambda \in \mathbb{R}\right\}$ and a magnitude $M_{0}=\|\boldsymbol{\omega}\|$. Therefore the control law (18) stabilizes all the particles to a relative equilibrium whose pitch is arbitrary. To reduce the dimension of the equilibrium set we combine the Lyapunov function (14) with the potential

$$
W(\boldsymbol{x}, \boldsymbol{\omega})=\sum_{k=1}^{N}\left(<\boldsymbol{\omega}, \boldsymbol{x}_{k}>-\alpha\right)^{2}, \quad \alpha \in[0,1),
$$

that is minimum when all the particles attain the same pitch $p_{0}=\alpha /\|\boldsymbol{\omega}\|^{2}$. This leads to the control law

$$
\begin{aligned}
\boldsymbol{u}_{k}= & R_{k}^{T}\left[\boldsymbol{\omega}+\left[\left(\boldsymbol{r}_{k}-\boldsymbol{r}_{\mathrm{av}}\right) \times \boldsymbol{\omega}-\boldsymbol{x}_{\mathrm{av}}\right.\right. \\
& \left.\left.+\left(<\boldsymbol{\omega}, \boldsymbol{x}_{k}>-\alpha\right) \boldsymbol{\omega}\right] \times \boldsymbol{x}_{k}\right] .
\end{aligned}
$$

The control law (25) stabilizes all the particles to a relative equilibrium whose magnitude and pitch are fixed by the design parameters $\alpha$ and $\|\boldsymbol{\omega}\|$. In particular, acting on $\alpha$ it is possible to separate circular equilibria $(\alpha=0)$ from helical equilibria $(\alpha \in(0,1))$. The global convergence analysis follows the lines of the proof of Theorem 1 and is omitted due to space constraints.

It is worth noting that when $\boldsymbol{\omega}$ is set to zero the control law (18) reduces to

$$
\boldsymbol{u}_{k}=R_{k}^{T}\left(\boldsymbol{x}_{k} \times \boldsymbol{x}_{\mathrm{av}}\right) .
$$

This control law stabilizes parallel formations and has been studied previously in [10].

\section{B. Stabilization about a consensus axis of rotation}

Because the control laws (18) and (25) depend on the vector $\omega$, the resulting closed-loop vector field is not invariant under an action of the symmetry group $S E(3)$. An important consequence is that additional information is required besides the relative configurations among the particles. To overcome this obstacle we propose a consensus approach to reach an agreement about the direction of the axis of rotation. We provide each particle with a consensus variable $\boldsymbol{\omega}_{k}$, and we denote by $\boldsymbol{\omega}_{k}^{a}=R_{k} \boldsymbol{\omega}_{k}$ the same quantity expressed in a (common) spatial reference frame. The potential

$$
\Omega\left(\boldsymbol{\omega}^{a}\right)=\frac{N}{2} \sum_{k=1}^{N}\left\|\boldsymbol{\omega}_{k}^{a}-\boldsymbol{\omega}_{\mathrm{av}}^{a}\right\|^{2},
$$

where $\boldsymbol{\omega}^{a}$ is the stacking vector of the vectors $\boldsymbol{\omega}_{1}^{a} \ldots, \omega_{N}^{a}$, decreases along the gradient dynamics

$$
\dot{\boldsymbol{\omega}}_{k}^{a}=\sum_{j=1}^{N} \boldsymbol{\omega}_{j}^{a}-\boldsymbol{\omega}_{k}^{a} .
$$

Expressing (28) in the body reference frame we obtain

$$
\dot{\boldsymbol{\omega}}_{k}=\hat{\boldsymbol{u}}_{k}^{T} \boldsymbol{\omega}_{k}+\sum_{j=1}^{N} R_{k}^{T} R_{j} \boldsymbol{\omega}_{j}-\boldsymbol{\omega}_{k},
$$

and we observe that the dynamics (29) are invariant under an action of the symmetry group $S O(3)$. It turns out that the dynamic control law resulting from the coupling between the consensus dynamics (29) with the control law (18) or (25) leads to a stabilizing (shape) control law.

Theorem 2: Consider model (2) equipped with the dynamic control law

$$
\begin{aligned}
\boldsymbol{u}_{k} & =\boldsymbol{\omega}_{k}+\left[R_{k}^{T}\left(\boldsymbol{r}_{k}-\boldsymbol{r}_{\mathrm{av}}\right) \times \boldsymbol{\omega}_{k}-R_{k}^{T} \boldsymbol{x}_{\mathrm{av}}\right] \times \boldsymbol{e}_{1}, \\
\dot{\boldsymbol{\omega}}_{k} & =\hat{\boldsymbol{u}}_{k}^{T} \boldsymbol{\omega}_{k}+\sum_{j=1}^{N} R_{k}^{T} R_{j} \boldsymbol{\omega}_{j}-\boldsymbol{\omega}_{k} .
\end{aligned}
$$

where $\boldsymbol{\omega}_{k}(0), k=1, \ldots, N$ are randomly selected.

The resulting closed-loop vector field is invariant under an action of $S E(3)$. All the solutions converge to an equilibrium of (19). The only asymptotically stable set is the set of relative equilibria of (2) and every other equilibrium is unstable.

Proof:(Sketch) To show that the resulting closed-loop vector field is invariant under an action of $S E(3)$, it is sufficient to observe that the dynamic control law (30) depends only on the relative orientations and relative positions of the particles. With the change of variables $\boldsymbol{\omega}_{k}^{a}=R_{k} \boldsymbol{\omega}_{k}$ (30) rewrites to

$$
\begin{aligned}
\boldsymbol{u}_{k} & =R_{k}^{T}\left(\boldsymbol{\omega}_{k}^{a}+\left[\left(\boldsymbol{r}_{k}-\boldsymbol{r}_{\mathrm{av}}\right) \times \boldsymbol{\omega}_{k}^{a}-\boldsymbol{x}_{\mathrm{av}}\right] \times \boldsymbol{x}_{k}\right), \\
\dot{\boldsymbol{\omega}}_{k}^{a} & =\sum_{j=1}^{N} \boldsymbol{\omega}_{j}^{a}-\boldsymbol{\omega}_{k}^{a} .
\end{aligned}
$$

We observe that (32) is independent from the particles dynamics. Therefore the solutions of (32) will converge to a consensus value $\overline{\boldsymbol{\omega}}$, i.e. $\boldsymbol{\omega}_{k}^{a} \rightarrow \overline{\boldsymbol{\omega}}$ when $t \rightarrow \infty$, for every $k$ for some $\overline{\boldsymbol{\omega}}$. Therefore (31) will asymptotically converge to

$$
\boldsymbol{u}_{k}=R_{k}^{T}\left(\overline{\boldsymbol{\omega}}+\left[\left(\boldsymbol{r}_{k}-\boldsymbol{r}_{\mathrm{av}}\right) \times \overline{\boldsymbol{\omega}}-\boldsymbol{x}_{\mathrm{av}}\right] \times \boldsymbol{x}_{k}\right)
$$

and system (2), equipped with the control law (31), will converge to an autonomous closed-loop system whose positive limit set is characterized in Theorem 1. Solutions are known to converge to a chain recurrent set of the limiting (autonomous) system [13]. The only chain recurrent sets of the limiting system are equilibria of (19). Relative equilibria of (2) are asymptotically stable and every other equilibrium is unstable (the details are omitted due to space constraints).

Remark 2: The control law (30) is the "dynamic" version of the control law (18) and therefore stabilizes all the particles to a relative equilibrium whose pitch is arbitrary. 
To fix the pitch to a desired value it is sufficient to derive the dynamic version of (25).

The control laws proposed in this section stabilize the relative equilibria of (2) under the assumption of all-toall communication among the particles. In the next Section we relax this assumption by substituting the quantities in (30) that require global information with consensus variables obeying consensus dynamics.

\section{StabilizATION OF RELATIVE EQUiLIBRIA IN THE PRESENCE OF LIMITED COMMUNICATION}

Consider the control law (30). By following the approach presented in [6] we substitute the quantities that require allto-all communication, i.e. $\boldsymbol{r}_{\mathrm{av}}$ and $\boldsymbol{x}_{\mathrm{av}}$, by local consensus variables. This leads to a generalization of the control law (30) to uniformly connected communication graphs ${ }^{1}$.

Theorem 3: Let $G(t)$ be a directed and uniformly connected communication graph and $L(t)$ the corresponding bounded and piecewise continuous Laplacian matrix ${ }^{2}$. Consider model (2) equipped with the control law

$$
\begin{aligned}
\boldsymbol{u}_{k} & =\boldsymbol{\omega}_{k}+\left(\boldsymbol{\omega}_{k} \times \boldsymbol{c}_{k}-\boldsymbol{b}_{k}\right) \times \boldsymbol{e}_{1} \\
\dot{\boldsymbol{\omega}}_{k} & =\hat{\boldsymbol{u}}_{k}^{T} \boldsymbol{\omega}_{k}-\sum_{j=1}^{N} L_{k j} R_{k}^{T} R_{j} \boldsymbol{\omega}_{j} \\
\dot{\boldsymbol{b}}_{k} & =\hat{\boldsymbol{u}}_{k}^{T} \boldsymbol{b}_{k}-\sum_{j=1}^{N} L_{k j} R_{k}^{T} R_{j} \boldsymbol{b}_{j} \\
\dot{\boldsymbol{c}}_{k} & =\hat{\boldsymbol{u}}_{k}^{T} \boldsymbol{c}_{k}-\boldsymbol{e}_{1}-\sum_{j=1}^{N} L_{k j} R_{k}^{T} R_{j} \boldsymbol{c}_{j}-\sum_{j=1}^{N} L_{k j} R_{k}^{T} \boldsymbol{r}_{j}
\end{aligned}
$$

where the initial conditions of the consensus variables are randomly selected. The resulting closed-loop vector field is invariant under an action of $S E(3)$. The only asymptotically stable set in the shape space is the set of relative equilibria of model (2). Every other equilibrium is unstable.

Proof:(Sketch) Observe that with the change of variables $\boldsymbol{\omega}_{k}^{a}=R_{k} \boldsymbol{\omega}_{k}, \boldsymbol{b}_{k}^{a}=R_{k} \boldsymbol{b}_{k}, \boldsymbol{c}_{k}^{a}=R_{k} \boldsymbol{c}_{k}+\boldsymbol{r}_{k}$ (33) rewrites to

$$
\begin{aligned}
\boldsymbol{u}_{k} & =R_{k}^{T}\left(\omega_{k}+\left[\left(\boldsymbol{r}_{k}-\boldsymbol{c}_{k}^{a}\right) \times \boldsymbol{\omega}_{k}^{a}-\boldsymbol{b}_{k}^{a}\right] \times \boldsymbol{x}_{k}\right. \\
\dot{\boldsymbol{\omega}}_{k}^{a} & =-\sum_{j=1}^{N} L_{k j} \boldsymbol{\omega}_{j}^{a} \\
\dot{\boldsymbol{b}}_{k}^{a} & =-\sum_{j=1}^{N} L_{k j} \boldsymbol{b}_{j}^{a} \\
\dot{\boldsymbol{c}}_{k}^{a} & =-\sum_{j=1}^{N} L_{k j} \boldsymbol{c}_{j}^{a}
\end{aligned}
$$

and the consensus dynamics are not influenced by the particles dynamics. Therefore, from Theorem 1 in [14], we conclude that the variables $\boldsymbol{\omega}_{k}^{a}, \boldsymbol{b}_{k}^{a}$ and $\boldsymbol{c}_{k}^{a}$ asymptotically converge to the consensus values $\overline{\boldsymbol{\omega}}, \overline{\boldsymbol{b}}$ and $\overline{\boldsymbol{c}}$ respectively.

The rest of the proof follows the same lines of the proof of Theorem (2) and therefore is omitted.

\footnotetext{
${ }^{1}$ A Graph $G(t)$ is said to be uniformly connected if there exists a time horizon $T>0$ and a node $k$ such that for all $t$ there exists a (directed) path from each node to the node $k$ in $\tilde{G}(t, t+T) \triangleq \bigcup_{t \in[t, t+T]} G(t)$.

${ }^{2}$ The graph Laplacian $L$ associated to the graph $G$ is defined as

$$
L_{k j}= \begin{cases}\sum_{i} a_{k i}, & j=k \\ -a_{k j}, & j \neq k\end{cases}
$$
}

where $a_{k j}$ are the elements of the adjacency matrix.
It is important to note that the control law (33) does not require all-to-all communication among the particles. In particular the convergence properties of Theorem 2 are here recovered in the presence of limited communication, for directed, time-varying (but uniformly connected) communication topologies. The same extension discussed in Remark 2 also applies to Theorem 3.

\section{CONCLUSION}

We proposed a methodology to stabilize relative equilibria in a model of identical, steered particles moving in threedimensional Euclidean space. Observing that the relative equilibria can be characterized by suitable invariant quantities, we formulated the stabilization problem as a consensus one. The formulation leads to a natural choice for the Lyapunov functions. Dynamic control laws are derived to stabilize relative equilibria in the presence of all-to-all communication and are generalized to deal with unidirectional and time-varying communication topologies. It is of interest (in particular from the application point of view) to study in the future how to reduce the dimension of the equilibrium set by breaking the symmetry of the proposed control laws.

\section{REFERENCES}

[1] N. Leonard, D. Paley, F. Lekien, R. Sepulchre, D. Fratantoni, and R. Davis, "Collective motion, sensor networks and ocean sampling," Proceedings of the IEEE, vol. 95, no. 1, pp. 48-74, 2007.

[2] J. Cortes, S. Martinez, T. Karatas, and F. Bullo, "Coverage control for mobile sensing networks," IEEE Trans. on Robotics and Automation, vol. 20, no. 2, pp. 243-255, 2004.

[3] E. W. Justh and P. S. Krishnaprasad, "Natural frames and interacting particles in three dimensions," in Proceedings of the 44th IEEE Conference on Decision and Control and European Control Conference, Seville, Spain, 2005, pp. 2841-2846.

[4] J. Fax and R. Murray, "Information flow and cooperative control of vehicle formations," IEEE Trans. on Automatic Control, vol. 49, no. 9, pp. 1465-1476, 2004.

[5] I. Couzin, J. Krause, R. James, G. Ruxton, and N. Franks, "Collective memory and spatial sorting in animal groups," J. Theor. Biol., vol. 218, pp. 1-11, 2002.

[6] L. Scardovi, A. Sarlette, and R. Sepulchre, "Synchronization and balancing on the $N$-torus," Systems and Control Letters, vol. 56, no. 5, pp. 335-341, 2007.

[7] L. Scardovi and R. Sepulchre, "Collective optimization over average quantities," in Proceedings of the 45th IEEE Conference on Decision and Control, San Diego, Ca, 2006, pp. 3369-3374.

[8] R. Sepulchre, D. Paley, and N. Leonard, "Stabilization of planar collective motion: All-to-all communication," IEEE Trans. on Automatic Control, vol. 52, no. 5, pp. 811-824, 2007.

[9] _ - "Stabilization of planar collective motion with limited communication," IEEE Trans. on Automatic Control, 2007, accepted.

[10] L. Scardovi, R. Sepulchre, and N. Leonard, "Stabilization laws for collective motion in three dimensions," in European Control Conference, Kos, Greece, 2007, pp. 4591-4597.

[11] A. Sarlette, R. Sepulchre, and N. Leonard, "Autonomous rigid body attitude synchronization," in Proceedings of the 46th IEEE Conference on Decision and Control, 2007, to appear.

[12] R. Murray, Z. Li, and S. S. Sastry, A Mathematical Introduction To Robotic Manipulation. CRC Press, 1994.

[13] K. Mischaikow, H. Smith, and H. Thieme, "Asymptotically autonomous semiflows; chain recurrence and lyapunov functions," Trans. of the American Mathematical Society, vol. 347, no. 5, pp. 1669-1685, 1995.

[14] L. Moreau, "Stability of continuous-time distributed consensus algorithms," in Proceedings of the 43rd IEEE Conference on Decision and Control, Paradise Island, Bahamas, 2004, pp. 3998-4003. 